\title{
Effects of Core Softness and Bimodularity of Fibreglass Layers on Flexural Stiffness of Polymer Sandwich Structures
}

\author{
Oldřich Šuba ${ }^{1, *}$, Oldřich Šuba jr. ${ }^{1}$, and Soňa Rusnáková $^{1}$ \\ ${ }^{1}$ Tomas Bata University in Zlín, Faculty of Technology, Department of Production Engineering, \\ Vavrečkova 275, Zlín, 760 01, Czech Republic,
}

\begin{abstract}
This paper deals with the study of the flexural stiffness of the sandwich structures based on fibreglass and polymeric foams. The influence of geometrical and material parameters on the resulting effective flexural stiffness of the sandwich structure is being studied experimentally, analytically and by using FEM models. The effective modulus of elasticity of the sandwich-structured element is being studied and its theoretical and model dependencies on the flexibility of the foam core and bimodularity of the fibreglass layers are being investigated. The achieved results are compared with the experimentally observed values. This study shows that it is necessary to pay special attention to the issue of flexural stiffness of the walls when designing sandwich shell products in order to prevent possible failures in the practical applications of these types of structures.
\end{abstract}

\section{Introduction}

The production technology along with the description of the characteristics of the sandwich structures on polymer base achieve sustainable rise and are the subjects of various research. Technological process is relatively demanding but the resulting structure is characterised by the high flexural stiffness and load capacity at very low weight [1-5].

Particularity of the mechanical behaviour of the sandwich structures comes along with certain singularities and difficulties at the design compared to the standard structures. Regarding the standard - homogenous plastic or fibreglass structures, the ratio of stiffness to strength is considerably low; therefore in many cases the product design is done by limiting conditions in relation with the wall stiffness and not with its strength. In case of load where there is pressure membrane stress in the shell wall, the design is based on the limiting conditions of the loss of the stability of the structure shell which are related with the value of flexural stiffness of the wall. In such cases the sandwich alternatives are applied [6-8].

On the other hand, the sandwich component concept brings along also some problems related to its macrostructure [4, 6-7]. The result of the geometrical and material parameters of the sandwich wall is the layered and thus non-homogeneous element whose aspects of its

*Corresponding author: $\underline{\text { suba@ft.utb.cz }}$ 
mechanical behaviour can be significantly different when compared to the conventional homogenous wall.

\section{Flexural Stiffness of Sandwich Structures. Effective Modulus of Elasticity}

For the pure flexural stiffness of the layered - composite cross section (excluded the effect of the core shear deformation), the modification of the technical theory of flexure can be expressed by

$$
K_{0}=E_{1+} J_{\mathrm{R}}
$$

where $E_{1+}$ is the modulus of elasticity of the surface layer of the fibreglass layer tensile value, $J_{R}$ represents quadratic moment of the reduced cross sectional area of the layered structure to the neutral axis - see Fig.1. So-called reduced cross sectional area of the nonhomogeneous (composite) element is generally given by the individual layers width reduction to the selected referential (usually the biggest) value of the modulus of elasticity of the given structure. The Fig.1 calculates with the possibility of including the bimodularity of the compressive layers, i.e. Different values of modulus of elasticity in tension $\mathrm{E}_{1+}$ and compression $\mathrm{E}_{1}$, when calculating the flexure stiffness.

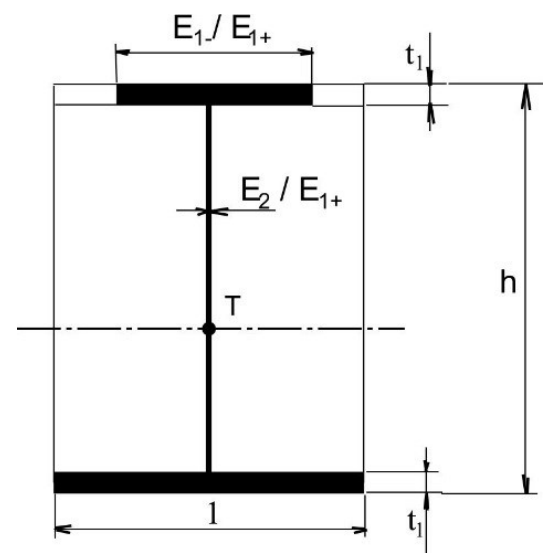

Fig. 1. Reduced cross-sectional area of the three-layered (sandwich) wall.

For the cases of practical evaluation of the experimental results, the concept of the effective modulus of elasticity of the sandwich structure was introduced. It is defined as the modulus of elasticity of theoretically homogenous (one-layered) wall thickness equal to the sandwich thickness $h$, having the same value of the flexure stiffness as the sandwich structure. Based on this condition, then according to (1)

$$
E_{e f}=E_{1+} \frac{12 J_{R}}{b h^{3}}
$$

The value of the „modulus of elasticity in flexure“ resulting from the standard bending test is apparently the experimentally given value of the effective modulus of elasticity of the sandwich wall. 


\section{Test: The effect of core shear compliance on the effective mechanical behaviour of sandwich elements}

One of the aspects of the mechanical behaviour of the sandwich structure is the effect of the generally significant shear compliance, i.e. low values of shear modulus of elasticity of the lighter core on the resulting effective mechanical behaviour of the sandwich element. As it is shown schematically in Fig. 2., the overall deformation of the sample in the three-point bending test is done by not only the deformation due to the impact of the flexural - normal stress but also by the relatively high contribution of the shear deformation as the shear stress given by the cross forces in the cross-sectional areas of the sandwich element are, as known, transmitted only by the the layer of the light-weight core.

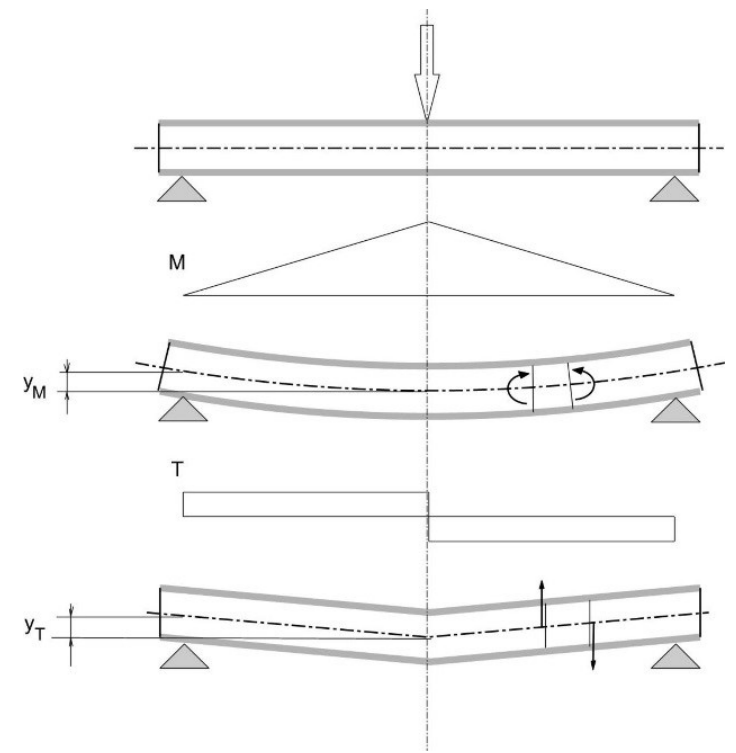

Fig. 2. Impact of core shear compliance on the overall deformation of the sandwich beam in the threepoint bending test.

The equation (1), or (2) respectively, including only the deformation component by bending stress in the compressive surface layers gives, unlike to the homogenous cross sections, show always higher values of the stiffness when compared to the values resulting from the experimental three-point standard test. Analytical expression of the deformation, or stiffness respectively, according to the three-point test where the shear compliance of the core is included, is according to the Fig. 2 with application of the principle of energy given by

$$
y_{M}+y_{T}=\frac{\partial A}{\partial F}
$$

where work deformation $A$ is given by the sum of the work deformation of the flexure moments and shear forces.

\section{Experimental Testing of Sandwich Structures Stiffness}

To verify the results of the numerical modelling, the sandwich structure with the outer coating of the fibreglass pre-impregnated fabric weighting $296 \mathrm{~g} / \mathrm{m}^{2}$ was used. As 
impregnating component of the prepreg the phenolic resin in amount of 42 weight percentage was applied. The core of the structures was made from polyethylene terephftalate (PET) foam with density of $100 \mathrm{~kg} / \mathrm{m}^{3}$, and the compression modulus of elasticity of $85 \mathrm{MPa}$.

The above-mentioned materials were used to produce the sandwich structures composed of one layer of prepreg from both, the upper and lower sides, and $20 \mathrm{~mm}$ thick PET core. It was produced by the method of the resin curing in the prepreg under the flexible foil in the curing own. Curing started by gradual heating for 30 mins to the temperature of $130^{\circ} \mathrm{C}$, followed by dwelling at this temperature for 120 mins.

After removing from the mould, the sandwich panels were cut into testing samples for standard bending test. There were done both, three-point and four-point tests. Testing speed was set on $5 \mathrm{~mm} / \mathrm{min}$ as recommended by the ASTM C393 standard.

\section{Numeric Modelling of Sandwich Structure Bending tests}

To study the dependency of the flexure stiffness of the sandwich wall on the stiffness of the weight-lighted core, the linear-elastic FEM models of the bending tests with the structures corresponding to the ones of the samples prepared for the bending tests were used.

The solution was done as linear-elastic analysis of the symmetrical half of the model for the three-point, or four-point bending test respectively. The FEM model for the three-point test is shown in Fig.3.

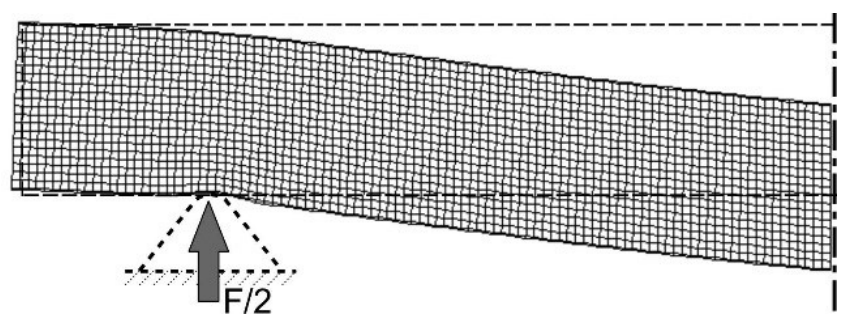

Fig. 3. Schema of the design model of the three-point bending test for the testing sample of the $20 \mathrm{~mm}$ sandwich structure with the core module of elasticity of $E_{2}=85 \mathrm{MPa}$ and the supports distance of $\mathrm{L}$ $=150 \mathrm{~mm}$. The character of the mesh deformation shows substantial proportion of the core shear compliance on the overall deformation of the sample.

\section{The Resulting Stiffness of Sandwich Structure Determined by Three-point and Four-point tests}

Comparison of the values obtained by model, analysis and experiment for the effective modulus of elasticity for the three-point test of the studied structure is shown in diagram in Fig. 4.

The effective modulus of elasticity values are taken for the coefficient of elasticity of covers in tension of $22000 \mathrm{MPa}$, which is determined by the manufacturer and it is, in fact, identical with the value obtained by the performed experiment.

It is obvious, that the dependencies $E_{\mathrm{ef}}$ determined analytically according to the equation (3) are located a bit under the values obtained by the FEM modelling. 


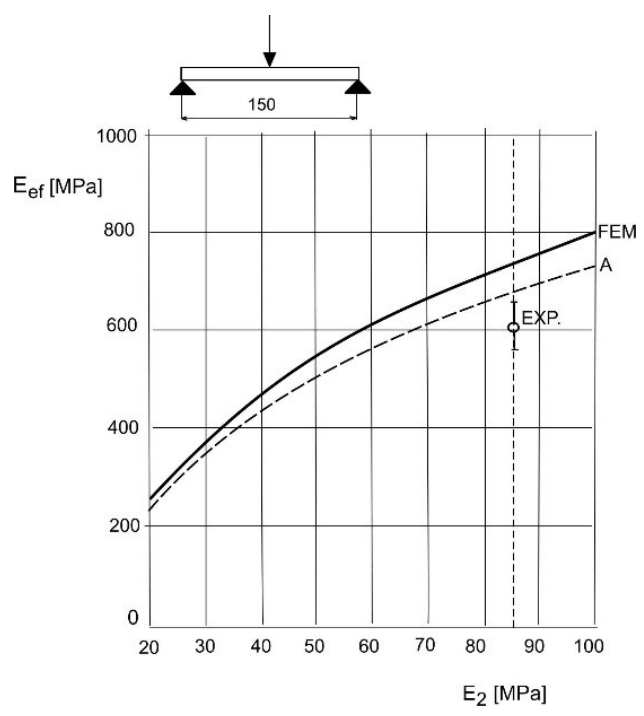

Fig. 4. Calculation dependencies for the effective modulus of elasticity during three-point testing on the foam core modulus of elasticity for the supports distance of $\mathrm{L}=150 \mathrm{~mm}$. Full curves - FEM, dashed curves - analytical calculation according to equation (3), EXP - range of experimentally obtained values $E_{\text {ef. }}$

The resulting differences between the experimentally obtained and numerical values of flexure stiffness of the studied structures can be attributed to the secondary aspects or imperfections respectively. Among those can be classified especially the problem of exact definition of the covers modulus of elasticity and their bimodularity, faults at determination of the shear modulus of core elasticity, as well as potential local indentation at the place of the supports. The impact of the core shear compliance is in the case of chosen $150 \mathrm{~mm}$ distance of the supports dominant. It will decline with the growing distance L between the supports and in case of the four-point test, it will be eliminated completely.

Comparison of the values obtained by model, analysis and experiment for the effective modulus of elasticity for the four-point bending test is shown in diagram in Fig.5.

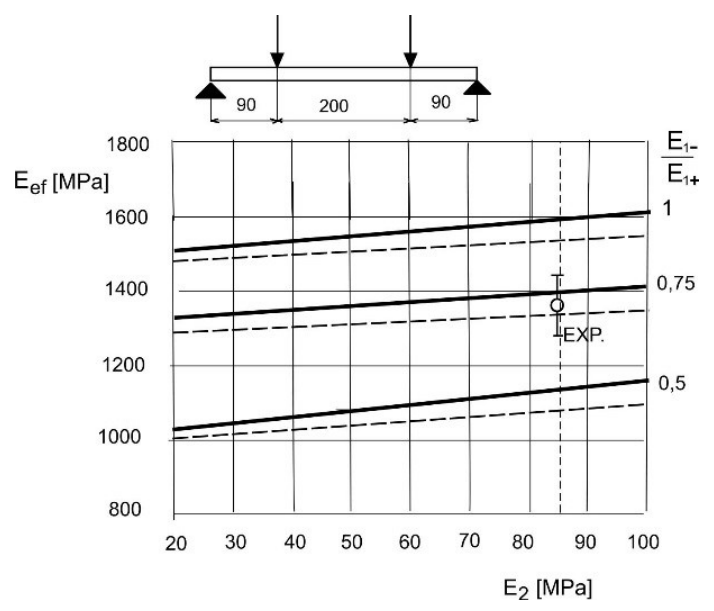

Fig. 5. Calculation dependencies of the effective modulus of elasticity during the four-point test on the modulus of elasticity of the foam core and bimodularity level of the compressive layers. Full curves - FEM, dashed curves - analytical calculation according to equation (3), EXP - range of experimentally obtained values $E_{\text {eff }}$ 
The results show somewhat surprising values; there is decrease of the flexure stiffness of the structure in the dependence on the bimodularity level of the compressive layers. Next, the calculated and experimentally obtained values of $E_{\text {ef }}$ are much higher in case of the four-point test when compared to the results of the three-point test. Regarding the studied structure, the pure flexure stiffness determined by the four-point test is approximately two times higher than the result of the three-point test with the same parameters. Experimental results point out certain bimodularity level of the studied samples.

\section{Conclusion}

The achieved results enable qualified evaluation of the effect of the core shear compliance, as well as of the bi-modularity level of the fibreglass compressive layer on the effective flexural stiffness of three-layered (sandwich) structures.

Based on the result of this study, it is necessary to pay attention to the design of sandwich products to prevent potential failure of these constructions in the practical applications.

Acknowledgement: This work and the project is realized with the financial support of the internal grant of TBU in Zlín No. IGA/FT/2017/002 funded from the resources of specific university research.

\section{References}

1. K. F. Karlsson, B. T. Åström. Applied Science and Manufacturing, 28, Issue 2 (1997)

2. M. Mohamed, S. Anandan, Z. Huo, V. Birman, J. Volz, K. Chandrashekhara. Composite Structures, 123 (2015)

3. J. R. Vinson. The Behavior of Sandwich Structures of Isotropic and Composite Materials (1st ed. New York: CRC Press, 1999)

4. D. Zenkert. The Handbook of Sandwich Construction, (Nordic Industrial Fund, Worcestershire, EMAS Publishing, 1997)

5. A. Zinno, E. Fusco, A. Prota, G. Manfredi. Composite Structures, 92 (2010)

6. G. Belingardi, M. P. Cavatorta, R. Duella. Composite Structures (2003)

7. L. Liu, H. Wang, Z. Guan. Composite Structures (2015)

8. M. Giglio, A. Gilioli, A. Manes. Computational Materials Science, 56 (2012)

9. Y-M. Jen, CH.-W. KO, H.-B. Lin. International Journal of Fatigue, 31, 3 (2009)

10. M. Garrido, J. R. Correia, T. Keller. Construction and Building Materials, 75 (2015)

11. B. Han, K.-K. Qin, B. Yu, Q.-CH. Zhang, CH.-Q. Chen, T. J. Lu. Composite Structures, 130 (2015) 